\title{
Evolution in Knee Replacement Implant
}

Saverio Comitini; Domenico Tigani; Danilo Leonetti; Matteo Commessatti; Federica Cuoghi; Paolo Barca; Antonio Martucci; Camilla Bettuzzi and Luca Amendola.

Department of Orthopaedics and Traumatology, Ospedale Maggiore, Bologna, Italy

\begin{abstract}
Major joint arthroplasty is undoubtedly one of the surgical success stories of modern times.

The first attempt of treating patients affected by knee osteoarthritis with arthroplasty goes back to the midnineteenth century.

In 1880 the German surgeon Themistocles Gluck implanted the first primitive hinge joints made of ivory.

The first part of the twentieth century saw the return of interpositional arthroplasty with the use of autologous tissues or metallic surfaces.

In the early 1960s, John Charnley's cemented metal-on-polyethylene total hip arthroplasty inspired the development of the modern total knee replacement.

He worked on the design of an implant that resurfaced the distal femur and proximal tibia without any direct mechanical link between the components began at the end of the sixties.

Technological developments in the field of knee replacement continue to increase the range of solutions for the recovery of joint mobility of painful knee arthritis.
\end{abstract}

Keywords: Condylar; Arthroplasty; Tibial; Knee

\section{Introduction}

The first idea of knee joint replacement dates back to 1880 in Berlin with the German surgeon Themistocles Gluck [1], who introduced, in a series of lectures an innovative system of joint replacement with a component made of ivory. The surgeon proposed to implant this in the bone with cement made of colophony, pumice and plaster of Paris. However, the only technique used during the first half of the last century was interpositional arthroplasty using autologous tissues or metallic surfaces [2]. During the late fifties and sixties knee arthroplasty was reconsidered and two different models were taken into consideration: constrained or hinged prosthesis or toward condylar replacement. Using the condylar replacement knee prosthesis the surgeon replaced the femoral and tibial load-bearing surface with nonlinked artificial components. In the late sixties at the Imperial College in London a study was conducted on the model of an implant without any direct mechanical link between the components that resurfaced the distal femur and proximal tibia. The original model, known as Freeman-Swanson [3] prosthesis, was none other than the metal "roller" component placed on the distal femur which articulated with a polyethylene tibial tray; nevertheless, it was necessary to perform the resection of both cruciate ligaments. In 1970 Peter Walker, John Insall, Chitranjan Ranawat and Alan Inglis at the Hospital for Special Surgery (HHS) of New York, developed the Duocondylar knee (Figure 1).

It was as a condylar, anatomical, symmetric and cemented knee that preserved both cruciate ligaments, without the anterior femoral flange. Another practical approach included the work by Averill and Khowayla with Howmedica (Howmedica, NY, U.S.) later developing the Geomedic knee, precursor for some of the anatomical knee models which allowed to preserve the cruciate ligaments.

This new design consisted in two spherical bearing surfaces creating a non- linked hinge-like mechanism. During the first experiences of these implants a high percentage of complications, such as component mobilization, malfunctioning of the components and infection, permitted the resurfacing of prosthesis development [4] and design in two different directions: the anatomical approach and the functional approach [5].

\section{Anatomical Approach}

This approach involved the implant that preserved most or all of the soft tissues of the knee joint, especially one or both cruciate ligaments. Yamamoto, in 1970 [6], at the Okayama University Medical School in Japan, was the first to make use of an anatomical femoral component with a minimally constrained single-piece polyethylene tibial component. The original model called the Kodama-Yamamoto knee, was made of COP alloy ( $\mathrm{Co}, \mathrm{Cr}, \mathrm{Ni}, \mathrm{Mo}, \mathrm{C}$, and $\mathrm{P}$ ) and consisted of an anatomical femoral mold component. This implant included an

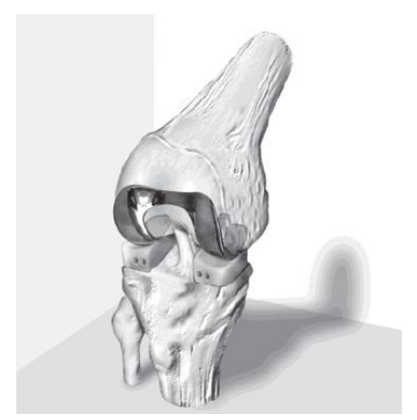

Figure 1: Duocondylar (courtesy of Prof. F. Catani)

${ }^{*}$ Corresponding author: Saverio Comitini, Department of Orthopaedics and Traumatology, Ospedale Maggiore, Bologna, Italy, Tel: +39 051647 8111; E-mail: saveriocomitini@gmail.com

Received December 29, 2014; Accepted April 23, 2015; Published April 2, 2014

Citation: Tigani D, Comitini S, Amendola L, Fosco M, Gentile P, et al., (2015) Evolution in Knee Replacement Implant. Single Cell Biol 4: 109. doi:10.4172/21689431.1000109

Copyright: $\odot 2015$ Tigani D, et al. This is an open-access article distributed under the terms of the Creative Commons Attribution License, which permits unrestricted use, distribution, and reproduction in any medium, provided the original author and source are credited. 
anterior femoral flange and a polyethylene tibial component with a central cutout for the preservation of both cruciate ligaments.

The same approach was followed by authors such as Waugh [7] from the University of California, Townley with the cemented Anatomical knee [8] and Sheedom who designed the Leeds knee [5].

All these prostheses had a horseshoe-shaped tibial component leaving a central and underlying gap for the preservation of both cruciate ligaments.

At the HSS in NY, during the early seventies, the Duocondylar knee was entirely modified in a new anatomical and symmetrical model: the Duopatellar knee (Figure 2).

Consequently an anterior femoral flange, patellar button, and a more dished tibial surface were added. The tibial component had a fixation peg, like the Total Condylar TC, the archetype of the functional approach, but an innovatively posterior rectangular central cutout, specifically designed for the retention of the PCL.

Even though the result of the Duopatella knee was extremely good at the HSS in NY, the posterior cruciate-preserving approach would be developed in Boston at the Robert Breck Brigham Hospital $[9,10]$.

In this important study institute, the model was modified: the medial tip of the femoral trochlear flange was eliminated, producing right and left patterns based on the asymmetry of the proximal femoral flange. This was done to adapt the component at the femoral shape of small female patients affected by rheumatoid arthritis and to reduce the medial overhang previously observed.

The posterior cruciate-sparing model of the Robert Brigham Hospital will later become the PFC knee (Cintor Division of Codman; later, Deputy, Johnson and Johnson).

Meanwhile, in June 1978 [5] Ewald implanted the Kinematic knee (Howmedica, NY, U.S.), which is the PCL-retaining version of the Duopatella knee, which was redesigned by Peter Walker, Clement Sledge and Fred Ewald.

The PCL-sparing version of the Kinematic II, Kinemax, and Kinemax Plus systems (Howmedica, NY, USA) will follow.

During the 80 's the surgical techniques and relative instrumentation of knee arthroplasty progressed significantly.

More specific instrumentation, later called Universal Instruments, was redesigned according to the recommendations of Kenna, Hungerford and Krakow.

These followed the rules of the anatomical concept of the measured resection technique rather than the more functional approach, which

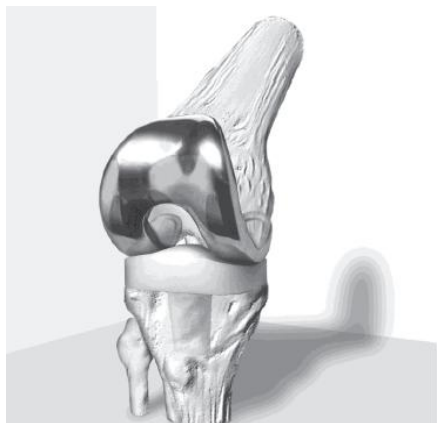

Figure 2: Duopatellar (courtesy of Prof. F. Catani). were used until then [3], obtaining equal and parallel flexion and extension gaps.

The characteristic of this new idea was to create, removing the bone and cartilage, an equal thickness for the prosthetic material.

Until this moment, the condylar total knee was primarily fixed with cement.

In 1980, Hungerford at Johns Hopkins [11], implanted the first Porous-Coated Anatomical Knee (PCA).

The implant was anatomical with asymmetric medial and lateral femoral condyles similar to the original models of Leeds and Townley.

It was the first time that a porous coating in a total condylar knee for cement-less fixation was introduced.

All three components were backed with metal and a 1.5-mm-thick sintered porous coating of cobalt chrome beads.

The Miller-Galante total knee, first implanted in 1986, was one of the first knee replacement predisposed for both fixation methods.

Because of its well-recognized biocompatibility titanium fibre composite was used for the bony ingrowth surface and the Titanium, Aluminium and Vanadium alloy (Ti6Al4V) was the innovative solution for the model of this prosthesis.

Therefore the implant is fixed to the tibial platform with titanium screws and pegs.

The cement-less patellar component consists of a metal-backed patella, fixed with fiber-mesh pegs.

Modularity of tibial polyethylene inserts were incorporated in order to allow better ligamentous balancing and the possibility of future isolated polyethylene replacement.

The cruciate retaining prosthesis was developed from the anatomical concept.

Some models consisted of a relatively flat surface on the sagittal and transversal plane (Kinemax e PCA) while others maintained a more congruent surface on the sagittal plane.

Some actual models inspired from this concept are: Genesis II (Smith Nephew Inc Warsaw, IN, U.S.), Duracon (Howmedica, NY, U.S.), Nexgen CR (Zimmer, Warsaw, IN, U.S.), and PFC CR (Deputy/ $\mathrm{J} \& \mathrm{~J})$.

\section{Functional Approach}

With the functional approach to knee arthroplasty the designers believed in, removing both cruciate ligaments, in order to streamline the knee biomechanics.

The first system, designed in 1973 at the HSS of New York [12], is the Total Condylar prosthesis (TC) consisting in two symmetric condylar surfaces and an articular polyethylene surface (Figure 3).

An aspect of this prosthetic model was a posterior decreasing radius of curvature and thus the components resulted perfectly congruent in extension and partially congruent in flexion

The TC knee would prove to have a high percentage of success, widely used, and would later demonstrates long survival [13].

However during the early period of its clinical use two concerns were highlighted. 


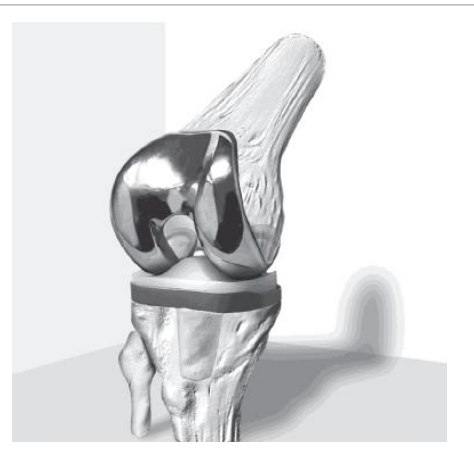

Figure 3: Total condylar (courtesy of Prof. F. Catani).

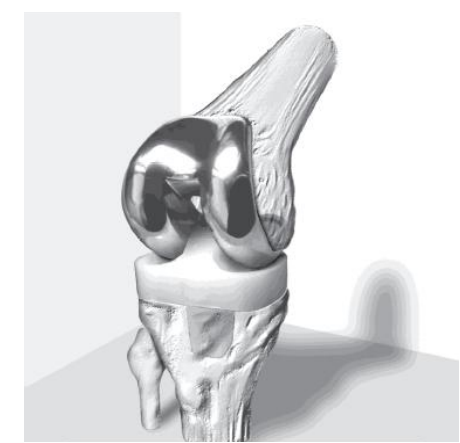

Figure 4: IB-I allpoly (courtesy of Prof. F. Catani)

First, the femoral component would shift forward, especially in flexion; in some cases this would even result in knee dislocation or belatedly in the loosening of the tibial component.

The second was the limited flexion achieved with the TC knee: maximally of $90^{\circ}$ degrees [5].

In 1978, Insall and Burstein, in order to overcome the above-mentioned problems, improved the original project with a cam mechanism, which reproduced a progressive rollback function of the PCL.

It was a tibial wedge-shaped post, which engaged an oval intercondylar femoral cam at $70^{\circ}$ degrees of flexion (Figure 4).

In 1978 at the HSS, Insall was the first to implant the IBPS knee.

The IBPS knee, thanks to improved flexion to $115^{\circ}$ and the elimination of the anterior femoral subluxation, was destined to become one of the most successful total condylar knee models [14].

In November 1980, the Insall-Burstein Modular knee (IBPS II) [15] was introduced: a metal-backed mono-block IBPS tibial component with direct-molded polyethylene.

The HSS posterior-stabilized knee model was the prototype for future development; in fact in 1988 the IBPS II knee (Zimmer, Warsaw IN USA) (Figure 5) made its first appearance, followed by the Optetrak Posterior-Stabilized knee (Exactech) (Figure 6) (in 1994 and then the Advance Posterior-Stabilized knee (Wright Mfg. Co, Memphis, TN).

The designers between the 1980s and the 1990s introduced many innovations in these functional knee models.

All of them, thanks to mechanical interaction between the femoral and tibial components, had the characteristic to produce their motion through a so-called guided motion.
Therefore, this meant that a specific movement, such as rollback, was directly related the aforementioned interaction.

The Kyocera Bi- Surface knee (Kyocera Corp, Kyoto, Japan) (Akagi et al., 2000), with moderately conforming bearing surfaces, during flexion behaved as a standard condylar replacement.

Beyond that, the load is transferred to a spherical surface protruding behind the femoral intercondylar region, found within a spherical depression at the posterior of the plastic tibial component.

The same concept of third condyle was already present in the model of the HLS prosthesis (Tornier S.A.S -France) developed in France by Dejour, Deschamps and Chambat. The first implant was performed in July 1984 [16].

The Medial Pivot knee (Wright Mfg. Co, Memphis, TN) is another example of guided motion knee.

Primarily the femoral component model consisted of a single radius curvature and a high level of conformity. The configuration of the medial compartment is similar to a ball and socket so that the medial side remains in the same position during flexion, but at the same time the lateral femoral condyle can displace behind. The purpose of the medial pivot model is to reproduce a more physiological kinematics. More recently, in contrast with this type of solution a new design has been created with a more concave lateral compartment which provides A/P stability similar to ACL deficient valgus knees: the 3D Knee. Therefore the lateral compartment is then fully congruent at $0^{\circ}$ and allows $15^{\circ}$ of axial rotation. During flexion, the knee has a

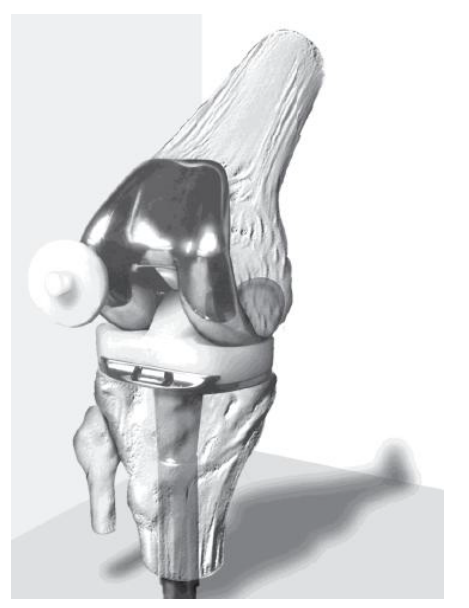

Figure 5: IB-II PS (courtesy of Prof. F. Catani).

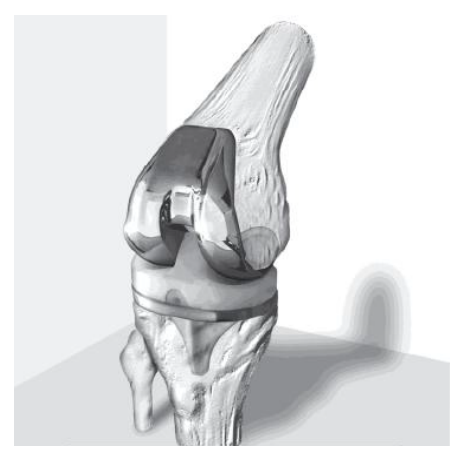

Figure 6: Optetrak (courtesy of Prof. F. Catani). 
Citation: Comitini S, Tigani D, Leonetti D, Commessatti M, Cuoghi F, et al. (2015) Evolution in Knee Replacement Implant. Single Cell Biol 4: 109. doi:10.4172/2168-9431.1000109

Page 4 of 7

greater range of femoral motion; this is possible because the concave lateral compartment controls it.

The aim of the 3D Knee is then to accommodate and control the cruciate deficient patterns of motions without constraint in stripe to reproduce the normal kinematic of the knee.

\section{Mobile bearing (MB) knee}

The collaboration at the New Jersey Medical School between Frederic Buechel, an orthopaedic surgeon, and Michael Pappas, a mechanical engineering professor was an innovative functional approach to condylar total knee design.

The aim of their model was to achieve a low polyethylene contact stress while maintaining knee flexion and avoiding overload of the implant bone interfaces. The original project started in 1977 [17] with the introduction of the Low Contact Stress (LCS) knee system: the first complete system approach to total knee replacement using meniscalbearing surfaces. The design specifically has the same spherical surface on the medio-lateral plane while it has, on the lateral side a decreasing curvature radius from extension to flexion. This form assures full area contact on the upper meniscal bearing from the $0^{\circ}$ to $45^{\circ}$ at which walking loads are encountered. The first LCS was designed and proposed as inclusive of both cruciate-sparing meniscal bearing total knee system.

Later the PCL-sacrificing rotating platform version became more widespread. Later on, many types of mobile bearing knees were developed by different designers and categorized according to their conformity: either partially or fully conforming.

Moreover the posterior stabilized MB represents a third group.

\section{Partially conforming MB}

The prototype of the partially conforming system is the second version of the LCS, consisting of a single plastic bearing that freely rotates about its post placed within a hole in the tibial tray.

In 1987 [3] Bourne and Rorabeck designed the Self Aligning MB (Sulzer Orthopedics, Austin, TX, U.S.), belongs to the partially conforming knees.

The characteristic feature of this prosthesis is an oval recess in posterior aspect of the polyethylene, which allowed unrestricted rotation and restricted AP translation about a tibial tray peg.

The mobile bearing knee model, called TACK (Waldemar Link $\mathrm{GmbH}$ \& Co. KG 1990), is characterized by the wide rotational movement thanks to the presence in the tibial tray of two semicircular guides that engage circular tracks on both sides of the polyethylene platform [3].

Another mobile bearing knee model was developed by Howmedica, the Interax Integrated Secure Asymmetric. This prosthesis has in extension nearly full conformity between the femoral component and tibial surface where, in flexion the conformity decreased gradually [3].

The tibial baseplate has two central posts that engage a curved, $\mathrm{t}$-shaped guide track within the meniscal bearing.

The Total Rotating Knee [3] (TRK) (Cremascoli Ortho S.p.A.,Verona, ITA) was designed by Professor F. Ghisellini.

The innovative design provided a central tibia post projecting from the centre of the tibial component.
Two types of the plastic inserts were available: the R type (Figure 7) which allowed freedom of rotation in case of PCL resection and the RS type (Figure 8).

The latter was indicated when the PCL was retained, allowing about $10 \mathrm{~mm}$ of antero-posterior sliding and more freedom of rotation.

\section{Fully conforming MB}

In 1986 Polyzoides and Tsakonas designed the Rotaglide Total knee System in Corin, (Corin and Cirencester, UK). This prosthesis, certainly progenitor of fully conforming MB knees, has a femoral shape with a constant radius of curvature and each condyle being part of a sphere of $24 \mathrm{~mm}$ radius: this allows that component congruency is retained throughout the range of flexion. The mobile meniscal bearing has two undercuts which permit up to $5 \mathrm{~mm}$ of anterior-posterior translation and $25^{\circ}$ of rotation, $12,5^{\circ}$ for each side. The tibial component has technical details that allow it to avoid anterior dislocation, hindered by an anterior bollard, while posterior dislocation is restricted by the rotation of the platform and the presence of another bollard in the middle of the tray.

In 1992 J Insall, P Aglietti and P Walker developed the Medially Biased Kinematics Knee (MBK-Zimmer) (Figure 9). The design

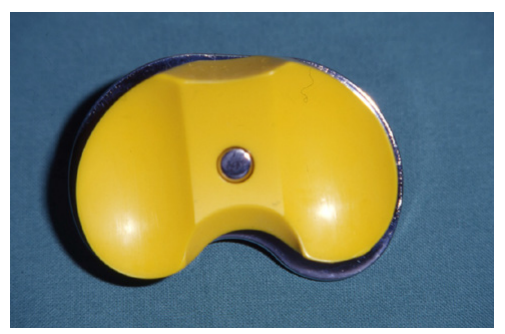

Figure 7: R type. Courtesy of Prof. F. Ghisellini.

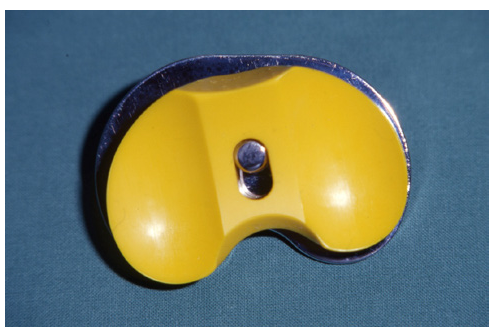

Figure 8: RS type. Courtesy of Prof. F. Ghisellini.

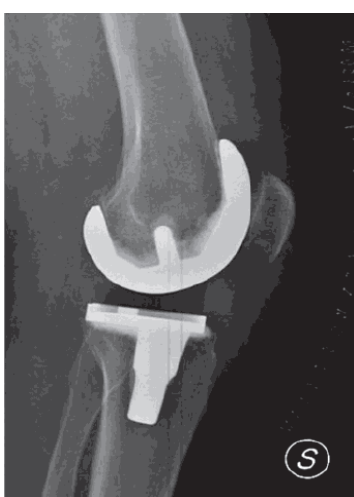

Figure 9: Multigen Plus total knee system (Lima-Lto). 
concept of this prosthesis has complete conformity at any degree of range of motion between the femoral component and the polyethylene insert, this especially during the flexion, rotation and AP translation of the tibial insert on the tibial tray. This design allows a medially biased kinematics guided by the natural knee's stronger medial structures and greater lateral mobility. The polyethylene has approximately 20 degrees of both internal and external rotation on the tibial baseplate about a D-shaped "mushroom" post. Moreover the tibial baseplate can translate antero - posteriorly of about $4,5 \mathrm{~mm}$.

An anterior stop prevents the plastic bearing from sliding off the tibial tray.

\section{Posterior stabilized MB}

The "cam and post" is the innovative feature of this category; the mechanism works on a polyethylene platform, which consisted in the presence of a cam located between the posterior femoral condyles that enters into contact working with a post projecting from the mobile rotating polyethylene insert.

The "cam and post" mechanism works as a third weight-bearing condyle to improve load transfer and minimize polyethylene stress.

In the years that followed, this mechanism inspired many designs such as the Two Radii Area Contact (TRAC, Biomet), which was introduced in 1997, the P.F.C. Sigma RPF (DePuy) and the LPS mobile Flex (Zimmer).

\section{High flexion knee and new materials}

With the start of the new millennium, many efforts have been made to improve the range of motion (ROM) that is an important issue in determining clinical outcome, and especially in the development of new materials. The indication of total knee arthroplasty has in fact extended to younger more active patients, and their demands and expectations have increased including secondary goals, other than pain relief, such as restoration of "normal-like" joint function, like running, playing tennis and downhill skiing all in order to suit their desired lifestyle [18].

Apart from being influenced by the condition of the patient and surgical technique, the final outcome, at least in part, depends on the implant design.

Modern TKAs designs accommodate knee mechanics in high flexion up to $155^{\circ}$. Several studies show a different kinematics between posterior cruciate ligament retaining designs and the posterior cruciate substituting prostheses [19]. PCL retaining design shows an erratic motion with potential for paradoxical roll forward [19].

The designer brings minimal but effective changes to the geometry of the traditional components to improve contact mechanics in the high-flexion ranges. In fact, these new high-flexion designs are not radically different from their traditional (not high-flexion designs) counterparts.

Regarding the sagittal geometry of the femoral component a reduction of the femoral condyles radii in the mid- and high-flexion ranges, if compared with the traditional implants, has shown some advantages.

In order to eliminate edge loading on the femoral component and on the posterior tibial articular surface, it was necessary to increase the area of the posterior femoral condyles. Furthermore this was made possible by restoring the posterior condylar offset, which has been previously emphasized as an important factor to improve flexion [20].
To clarify this concept the Authors demonstrated that for every $2 \mathrm{~mm}$ decrease in posterior condylar offset, the maximal obtainable flexion was reduced by a mean of $12.2^{\circ}$.

Designers choose mobile bearings for the reasoning that in order to obtain greater knee flexion more internal rotation of the tibia was necessary, which happens with extreme posterior shift of the lateral femoral condyle over the posterior tibial platform increased tibial rotation with deep flexion and the theoretical advantage of improved contact area $[21,22]$.

These changes are associated to a modified cam/post mechanism, which allow a more jump distance and avoids dislocation at deep flexion angles. High flex design prosthesis provides modification of the patella-femoral joint in order to accommodate high degree of flexion. For the most part, to reduce the contact stresses on the patella through a full range of motion, the design of femoral trochlear should be deeper and simultaneously the patella should glide smoothly. Also as it is easily observable the recent version of the tibial articular component has been recessed: this in order to reduce extensor mechanism impingement, especially during deep flexion.

As the goal of arthroplasty is that also to return to those their daily habits, and their own style of life, in 1989, in Japan the oriental lifestyle foresaw that people sit more often on the floor than on a chair; it was necessary to develop a more specific design, thus the KU knee (Kyoto University knee) was developed.

The exceptional aspect of this model is that it has an auxiliary joint of a ball and socket at the centre of the posterior part. This auxiliary joint, improves a rollback movement adding a rotational function when the flexion becomes greater; it represents a certain type of posteriorstabilized knee. This knee design was later called bisurface knee (BS knee) [23] because of its unique biphasic surface structure used for different purposes such as weight bearing and flexion movement. Another innovative feature of this prosthesis is the presence of zirconia ceramics $\left(\mathrm{ZrO}_{2}\right)$ for the femoral component. According to several studies further improvement in the longevity of the arthroplasty can be achieved with more durable bearing materials [24,25]. Throughout the years the designers have modified the component materials in order to improve the longevity of the implant. However, wear and duration of the implant are still a concern.

Despite the improvement in manufacturing and elimination of gamma-irradiation in air has already resulted in fewer wear-related problems, concern remains about the adhesive and abrasive wear caused by the hard counter face of the femoral component [25]. It has previously been shown that roughening of the cobalt-chromium (CoCr) alloy can potentiate wear of the PE [24] and this could be closely related to osteolysis, instability and loosening of the implants from the underlying bone. The designers are striving to find different alloys for femoral components, alternative to the classic CoCrMo one (Stellite TM), both for complete ceramic and for ceramic surfaces. Furthermore, due to the advantages of ceramic bearing surfaces in terms of superior lubrication, friction, and wear properties the surfaces in total joint arthroplasty, compared to cobalt-chrome alloy $(\mathrm{CoCr})$ are well recognized in studies. These prosthesis with the ceramic femoral components can be more useful in younger patient and those affected by allergies to metallic ions (Stellite) [26-29].

In total hip and total knee arthroplasty clinical studies $[26,30]$ demonstrated that ceramic bearings are associated with fewer wear particles that incite a less intense inflammatory host immune response than the metal-on-polyethylene articulations. 


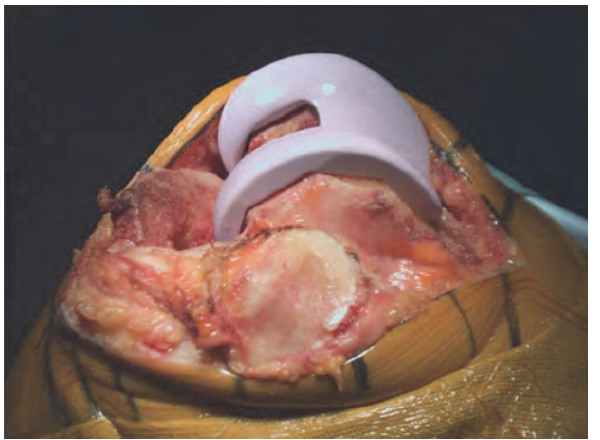

Figure 10: BIOLOX Delta ceramic femoral component.

But due to its brittle nature and inability to withstand high-impact tensile forces the ceramic material is of concern in clinical applications.

However, more than 10 years, long-term follow-up of different cemented ceramic knees were performed in Japan and showed satisfactory results with low rate fractures of ceramic component [23,31]. CeramTec (AG, Polchingen, Germany) has recently introduced in Europe the BIOLOX Delta. The abovementioned company has presented an important improvement using a composite matrix material containing $82 \%$ vol. alumina $\left(\mathrm{Al}_{2} \mathrm{O}_{3}\right)$ and $17 \%$ vol. zirconia $(\mathrm{ZrO} 2)$ that provide good mechanical characteristics in terms of strength and resistance [27]. Using this material it was possible to develop a femoral component with a tensile strength that meets the demands for application in TKA [32]. In order to evaluate the clinical and radiological outcomes after some in vitro tests $[33,34]$, a ceramic material was used for a prospective international multicentre study started in 2008.

In the following images system subject of the study: Multigen Plus total knee system (Lima-Lto) (Figure 10) with the new BIOLOX Delta ceramic femoral component (Figure 10).

In the U.S. an alternative strategy has been followed to decrease the PE wear, the component surface was subjected to the process of transformation of metal to oxidized zirconium and this method was applied for both THA and TKA. More precisely a wrought zirconium alloy (Zr-2.5\% Niobium) is oxidized by thermal diffusion to create a 5-mm oxidized zirconium layer [35].

Although existing data is encouraging with both strategies [36], further studies are necessary to define the indications and clinical outcomes of the use of ceramic surfaces in TKA.

\section{Conclusions and future perspective.}

TKA is an effective solution in the treatment of severe arthritis of the knee. The indications are increasingly extended to younger patients with higher functional demands. Some patients deterred by the negative experiences of people they know are demanding guarantees especially in terms of longevity. Therefore the surgeon, during the indication and choice of the prosthetic implant, must be supported by current data and multi-centre experience. Technological developments in the field of knee replacement continue to increase and particularly with focus on designing prosthesis which are more effective and tolerated by active patients.

This explains the considerable attention, first, to the morphological aspects of prosthetic components, and secondly, to the choice of materials with the aim of ensuring greater wears resistance and improved biocompatibility.

This is an indispensable condition for the stability of the prosthetic implant and for the success of the replacement.

\section{References}

1. Eynon-Lewis NJ, Ferry D, Pearse MF (1992) Themistocles Gluck: an unrecognised genius. BMJ 305: 1534-1536.

2. McKeever DC (1960) Tibial plateau prosthesis. Clin Orthop Rel Res 19: 3-12.

3. Freeman MA, Swanson SA, Todd RC (2003) Total replacement of the knee using the Freeman-Swanson knee prosthesis. 1973. Clin Orthop Relat Res : 4-21.

4. Insall J, Scott WN (2001) Surgery of the Knee (third edition), ChurchillLivingstone, New York.

5. Robinson RP (2005) The early innovators of today's resurfacing condylar knees. J Arthroplasty 20: 2-26.

6. Yamamoto S (1979) Total knee replacement with the Kodama-Yamamoto knee prosthesis. Clin Orthop Relat Res : 60-67.

7. Waugh TR, Smith RC, Orofino CF, Anzel SM (1973) Total knee replacement operative technic and preliminary results. Clin Orthop Relat Res : 196-201.

8. Townley C, Hill L (1974) Total knee replacement. Am J Nurs 74: 1612-1617.

9. Ranawat CS, Sculco TP (1985) History of the development of total knee prosthesis at the Hospital for Special Surgery. In Total-Condylar Knee Arthroplasty Springer New York : 3-6.

10. Scott RD (1982) Duopatellar total knee replacement: the Brigham experience. Orthop Clin North Am 13: 89-102.

11. Hungerford DS, Kenna RV, Krackow KA (1982) The porous-coated anatomic total knee. Orthop Clin North Am 13: 103-122.

12. Insall J, Ranawat CS, Scott WN, Walker P (1976) Total condylar knee replacment: preliminary report. Clin Orthop Relat Res : 149-154.

13. Vince KG, Insall JN, Kelly MA (1989) The total condylar prosthesis. 10- to 12 year results of a cemented knee replacement. J Bone Joint Surg $\mathrm{Br}$ 71: 793797

14. Abdeen AR, Collen SR, Vince KG (2010) Fifteen-year to 19-year follow-up of the Insall-Burstein-1 total knee arthroplasty. J Arthroplasty 25: 173-178.

15. Stern SH, Insall JN (1992) Posterior stabilized prosthesis. Results after followup of nine to twelve years. J Bone Joint Surg Am 74: 980-986.

16. Dejour H, Deschamps G, Chambat $P$ (1989) The HLS prosthesis in total genu, $35^{\circ}$ einsegnement Booklet SOFCOT. Colletion led by J Duparc.

17. Buechel FF, Pappas MJ (1986) The New Jersey Low-Contact-Stress Knee Replacement System: biomechanical rationale and review of the first 123 cemented cases. Arch Orthop Trauma Surg 105: 197-204.

18. Noble PC, Conditt MA, Cook KF, Mathis KB (2006) The John Insall Award: Patient expectations affect satisfaction with total knee arthroplasty. Clin Orthop Relat Res 452: 35-43.

19. Dennis DA, Komistek RD, Stiehl JB, Walker SA, Dennis KN (1998) Range of motion after total knee arthroplasty: the effect of implant design and weightbearing conditions. J Arthroplasty 13: 748-752.

20. Bellemans J, Banks S, Victor J, Vandenneucker H, Moemans A (2002) Fluoroscopic analysis of the kinematics of deep flexion in total knee arthroplasty. Influence of posterior condylar offset. J Bone Joint Surg Br 84: 50-53.

21. Kurosaka M, Yoshiya S, Mizuno K, Yamamoto T (2002) Maximizing flexion after total knee arthroplasty: the need and the pitfalls. J Arthroplasty 17: 59-62.

22. Nakagawa S, Kadoya Y, Todo S, Kobayashi A, Sakamoto H, et al. (2000) Tibiofemoral movement 3: full flexion in the living knee studied by MRI. J Bone Joint Surg Br 82: 1199-1200.

23. Akagi M, Nakamura T, Matsusue Y, Ueo T, Nishijyo K, et al. (2000) The Bisurface total knee replacement: a unique design for flexion. Four-to-nine-year follow-up study. J Bone Joint Surg Am 82-82A: 1626-33.

24. White SE, Whiteside LA, McCarthy DS, Anthony M, Poggie RA (1994) 
Citation: Comitini S, Tigani D, Leonetti D, Commessatti M, Cuoghi F, et al. (2015) Evolution in Knee Replacement Implant. Single Cell Biol 4: 109. doi:10.4172/2168-9431.1000109

Simulated knee wear with cobalt chromium and oxidized zirconium knee femoral components. Clin Orthop Relat Res : 176-184.

25. Muratoglu OK, MarkA, Vittetoe DA, Harris WH, Rubash HE (2003) Polyethylene damage in total knees and use of highly crosslinked polyethylene. J Bone Joint Surg Am 85-85A Suppl 1: S7-S13.

26. Spector BM, Ries MD, Bourne RB, Sauer WS, Long M, et al. (2001) Wear performance of ultra-high molecular weight polyethylene on oxidized zirconium total knee femoral components. J Bone Joint Surg Am 83-83A Suppl 2 Pt 2 : 80-6.

27. Dalla Pria $P$ (2007) Evolution and new application of the alumina ceramics in joint replacement. Eur J Orthop Surg Traumatol 17: 253-256 .

28. Greenwald AS, Garino JP (2001) American Academy of Orthopaedic Surgeons Committee on Biomedical Engineering; American Academy of Orthopaedic Surgeons. Committee on Hip and Knee Arthritis. Alternative bearing surfaces: the good, the bad, and the ugly. J Bone Joint Surg Am 83-83A Suppl 2 Pt 2: 68-72.

29. Jacobs JJ, Shanbhag A, Glant TT, Black J, Galante JO (1994) Wear Debris in Total Joint Replacements. J Am Acad Orthop Surg 2: 212-220.

30. Mont MA, Booth RE Jr, Laskin RS, Stiehl JB, Ritter MA, et al. (2003) The spectrum of prosthesis design for primary total knee arthroplasty. Instr Course Lect 52: 397-407.
31. Koshino T, Okamoto R, Takagi T, Yamamoto K, Saito T (2002) Cemented ceramic YMCK total knee arthroplasty in patients with severe rheumatoid arthritis. J Arthroplasty 17: 1009-1015.

32. Kluess D, Souffrant R, Fritsche A, Mittelmeier W, Bader R (2009) Explicit Finite-Element-Analysis of the Impaction Behavior of a Ceramic Femoral Component in Total Knee Replacement. Proceedings 55th Annual Meeting of the Orthopaedic Research Society, Las Vegas.

33. Cristofolini L, Affatato S, Erani P, Tigani D, Viceconti M (2009) Implant fixation in knee replacement: preliminary in vitro comparison of ceramic and metal cemented femoral components. Knee 16: 101-108.

34. Bergschmidt P, Bader R, Ganzer D, Hauzeur C, Lohmann C, et al. (2012) Ceramic femoral components in total knee arthroplasty-two year follow-up results of an international prospective multi-centre study. The open orthopaedics journal 6: 172

35. Laskin RS (2003) An oxidized Zr ceramic surfaced femoral component for total knee arthroplasty. Clin Orthop Relat Res : 191-196.

36. Innocenti M, Civinini R, Carulli C, Matassi F, Villano M (2010) The 5-year results of an oxidized zirconium femoral component for TKA. Clin Orthop Relat Res 468: 1258-1263. 